\title{
Soft X-ray coronal spectra at low activity levels observed by RESIK
}

\author{
B. Sylwester ${ }^{1}$, J. Sylwester ${ }^{1}$, and K. J. H. Phillips ${ }^{2}$ \\ 1 Space Research Center, Polish Academy of Sciences, Kopernika 11, 51-622 Wrocław, Poland \\ e-mail: [bs;js]@cbk.pan.wroc.pl \\ 2 UCL-Mullard Space Science Laboratory, Holmbury St. Mary, Dorking, Surrey RH5 6NT, UK \\ e-mail: kjhp@mssl.ucl.ac.uk \\ Received 16 July 2009 / Accepted 1 February 2010
}

\section{ABSTRACT}

\begin{abstract}
Context. The quiet-Sun X-ray emission is important for deducing coronal heating mechanisms, but it has not been studied in detail since the Orbiting Solar Observatory (OSO) spacecraft era. Bragg crystal spectrometer X-ray observations have generally concentrated on flares and active regions. The high sensitivity of the RESIK (REntgenovsky Spectrometer s Izognutymi Kristalami) instrument on the CORONAS-F solar mission has enabled the X-ray emission from the quiet corona to be studied in a systematic way for the first time.

Aims. Our aim is to deduce the physical conditions of the non-flaring corona from RESIK line intensities in several spectral ranges using both isothermal and multithermal assumptions.

Methods. We selected and analyzed spectra in 312 quiet-Sun intervals in January and February 2003, sorting them into 5 groups according to activity level. For each group, the fluxes in selected spectral bands have been used to calculate values of parameters for the best-fit that leads to intensities characteristic of each group. We used both isothermal and multitemperature assumptions, the latter described by differential emission measure (DEM) distributions. RESIK spectra cover the wavelength range (3.3-6.1 Å). This includes emission lines of highly ionized $\mathrm{Si}, \mathrm{S}, \mathrm{Cl}, \mathrm{Ar}$, and $\mathrm{K}$, which are suitable for evaluating temperature and emission measure, were used.

Results. The RESIK spectra during these intervals of very low solar activity for the first time provide information on the temperature structure of the quiet corona. Although most of the emission seems to arise from plasma with a temperature between $2 \mathrm{MK}$ and $3 \mathrm{MK}$, there is also evidence of a hotter plasma $(T \sim 10 \mathrm{MK})$ with an emission measure 3 orders smaller than the cooler component. Neither coronal nor photospheric element abundances appear to describe the observed spectra satisfactorily.
\end{abstract}

Key words. Sun: X-rays, gamma rays - Sun: abundances - Sun: corona

\section{Introduction}

The RESIK X-ray spectrometer on the Russian CORONAS-F solar orbiting mission (circular polar orbit: altitude $550 \mathrm{~km}$, period $96 \mathrm{~min}$ ) obtained numerous high-resolution flare and active-region spectra in the 3.3-6.1 $\AA$ range over the period August 2001-May 2003. The RESIK instrument (Sylwester et al. 2005) was a bent crystal spectrometer with four spectral channels in which solar X-ray emission was diffracted by crystal wafers made of silicon ( $\mathrm{Si} 111,2 d=6.27 \AA$ ) and quartz (Qu 1010 $0,2 d=8.51 \AA)$. Although most previous spacecraft crystal spectrometers suffered from the strong instrumental backgrounds caused by fluorescence of the crystal material, RESIK had a system of pulse-height analyzers enabling primary solar photons to be distinguished from secondary photons produced by fluorescence. The background could thus be practically eliminated for much of the period 2003 January-March when the non flaring solar X-ray activity was often below $\mathrm{C} 1$ class as measured by the GOES $1-8 \AA$ sensor. The sensitivity of RESIK was maximized by not having a collimator placed in front of the crystals. This, like the equivalent Bragg Crystal Spectrometer on the Yohkoh spacecraft (operational 1991-2001), produced some spectral confusion when two simultaneous bright sources were present on the Sun, but in practice this rarely occurred. The low-activity corona gives rise to X-ray line profiles in RESIK spectra having large line widths through spatial broadening.

Several analyses have been done on spectra of solar flares from RESIK (Sylwester et al. 2006a; Chifor et al. 2007; Sylwester et al. 2008), but here we report on spectra obtained during a period of sustained low solar activity, which have high statistical quality because of the relatively high sensitivity of RESIK. The observed spectral shapes including continua are available for analysis of the temperature structure of the emitting regions, specifically the differential emission measure as a function of electron temperature $T$; and from this, the absolute abundances of the elements giving rise to the spectral lines can be assessed.

Quiet-Sun X-ray spectra have not received nearly as much attention in the past as those from flares, but some properties of the quiet Sun have been widely studied using its ultraviolet emission, which has been measured by the following experiments: Orbiting Solar Observatory OSO series (1962-1975; see for instance Dupree \& Reeves 1971; Dupree et al. 1973), Aerobee rocket spectrometer (1969, see Malinovsky \& Heroux 1973), 9 months of the Skylab mission (May 1973-February 1974, see Vernazza \& Reeves 1978), the series of 9 Solar EUV Rocket Telescope and Spectrograph (SERTS) flights (the first in 1989, see Brosius et al. 1996, 1998), the SOHO mission (starting in December 1995, CDS and SUMER data, see Warren et al. 1998). 
The data obtained have been used as well to construct a reference atlas of quiet-Sun ultraviolet radiation (Curdt et al. 2004) from which differential emission measure (DEM) distributions can be inferred. Brosius et al. (1996) calculated DEM distributions for quiet-Sun conditions during two observing periods near the maximum of Cycle 21 (1991 May 7 and 1993 August 17) based on SERTS data. Their DEM solutions included a power law in the temperature range $6.3 \times 10^{4} \mathrm{~K}-5.0 \times 10^{5} \mathrm{~K}$ with hot plasma as shown by a localized maximum at $5 \mathrm{MK}$. A DEM with similar distribution was obtained by Kretzschmar et al. (2004) based on SOHO SUMER data, having a power-law shape in the range $2.0 \times 10^{4}-2.0 \times 10^{5} \mathrm{~K}$ with a high-temperature bump at 1.1 MK. Ralchenko et al. (2007) have studied quiet-corona spectra as observed by SUMER during the 2000 June 13-19 period, deducing that the observed line intensities can be satisfactorily described by a model with two Maxwellian electron distributions, a first population corresponding to an isothermal temperature of $\sim 1.3 \times 10^{6} \mathrm{~K}$, and a second, smaller population $(\sim 5 \%)$ of hot (300-400 eV) electrons accounting for the intensities of highly charged $\mathrm{Ar}$ and $\mathrm{Ca}$ ion lines observed by SUMER. The DEM analysis was also performed using SOHO Coronal Diagnostic Spectrometer (CDS) data for the internetwork, network, and bright network regions of the quiet Sun by O'Shea et al. (2000). They find that the DEM distributions differ in each region over the temperature range $0.25-1 \mathrm{MK}$.

Recently, Young et al. (2007) have published a quiet-Sun extreme ultraviolet (EUV) spectrum in the ranges 170-211 $\AA$ and 246-292 $\AA$, as observed on 2006 December 23 by the Hinode EUV Imaging Spectrometer (EIS).

The quiet-Sun hard X-ray emission observed by RHESSI has been examined by Hannah et al. (2007) using a fan-beam modulation technique during seven periods of off-pointing of the RHESSI spacecraft between 2005 June and 2006 October. They established new upper limits on the 3-200 keV X-ray emission for when the GOES level of activity was below A1 class, updating much earlier measurements (Peterson et al. 1966). Schmelz et al. (2010) have investigated the emission of a nonflaring active region based on the ten filters of the Hinode X-ray Telescope data using two independent algorithms to reconstruct the differential emission measure distribution. In addition to the typical low-temperature emission measure $(T<5 \mathrm{MK})$, they find a very hot component $(\sim 30 \mathrm{MK})$ with small emission measure. These findings have recently been modified by Schmelz (2009, priv. comm.): the temperature of the hotter component is now much lower, around $10 \mathrm{MK}$. Reale et al. (2009) have investigated the Hinode XRT data averaged over one hour during a nonflaring period (2006 November 12), finding a hotter component (temperature $\sim 6.3 \mathrm{MK}$ ) corresponding to a nonflaring active region. These observational results are supported by the theoretical work of Klimchuk et al. (2008) who use hydrodynamic simulations of nanoflares to predict a small amount of hot plasma in addition to the dominant 2-3 MK plasma component.

The RESIK spectra recorded during solar minimum can bridge the gap between the results obtained from ultraviolet and X-ray images and spectra and the models of coronal plasma heating. In earlier work, we analyzed RESIK spectra to determine the conditions of flaring plasmas, but here we apply the same techniques of analyzing emission during low-level periods to deduce the properties of the quiet-Sun corona.

\section{RESIK spectra selection and isothermal analysis}

We selected 312 time intervals, each $5 \mathrm{~min}$ in duration, for which solar activity levels in the first quarter of 2003 were at

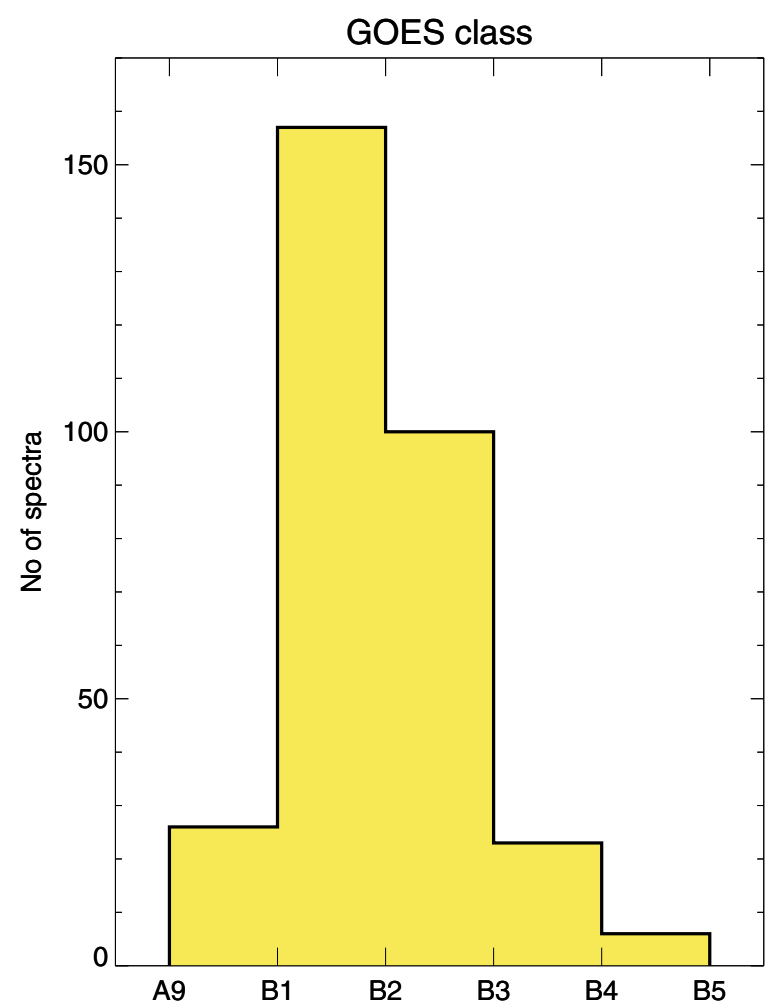

Fig. 1. Histogram showing the respective GOES activity for selected 312 time intervals (January-February 2003).

their lowest. The measurements were taken outside intervals of increased background owing to passages of the CORONAS-F spacecraft through polar ovals or the South Atlantic Anomaly. Early in 2003, the RESIK instrument characteristics (high voltage and amplitude discrimination) were set at their optimum values. Each spectrum analyzed was integrated over a $302 \mathrm{~s}$ interval, the longest selectable period for a low-flux operating mode, so the overall data accumulation time was $26.2 \mathrm{~h}$. The spectra correspond to GOES activity levels from $\sim \mathrm{A} 9$ to $\sim \mathrm{B} 5$. We found it useful to group the observed spectra according to the activity level at the time that they were recorded, so that the summed spectra for any group has an improved statistical quality. The division into groups was chosen according to activity level steps of 0.1 in the logarithm (0.1 dex).

In Fig. 1 we show the distribution of spectra with GOES classes. The lowest class includes spectra from the GOES class between A9 and B1 (1-8 $\AA$ flux from $9 \times 10^{-8}$ to $1 \times 10^{-7} \mathrm{~W} \mathrm{~m}^{-2}$ ), attained between 2003 February 23 and February 25. Although flaring periods were always carefully avoided in selecting spectra, it was impossible to avoid the presence of active regions, as RESIK operated close to the maximum of Cycle 23.

A typical pattern of activity during the time of the selected spectra collection is illustrated (Fig. 2) with a set of full-disk EUV images from the Extreme-ultraviolet Imaging Telescope (EIT; Delaboudiniere et al. 1995) aboard the Solar and Heliospheric Observatory (SOHO) on 2003 February 24 around 19:00 UT. The images are in the spectral bands $304 \AA$ (He II), $171 \AA$ (Fe IX-X), $195 \AA$ (Fe XII), and $284 \AA$ (Fe XV), which are sensitive to temperatures of about $80000 \mathrm{~K}, 1.3 \mathrm{MK}, 1.6 \mathrm{MK}$, and $2 \mathrm{MK}$, respectively. Weak active-region emission is visible in all passbands. This is also true for all other times when RESIK spectra were selected, so our results therefore apply to times when the corona was quiet and also when there were weak 


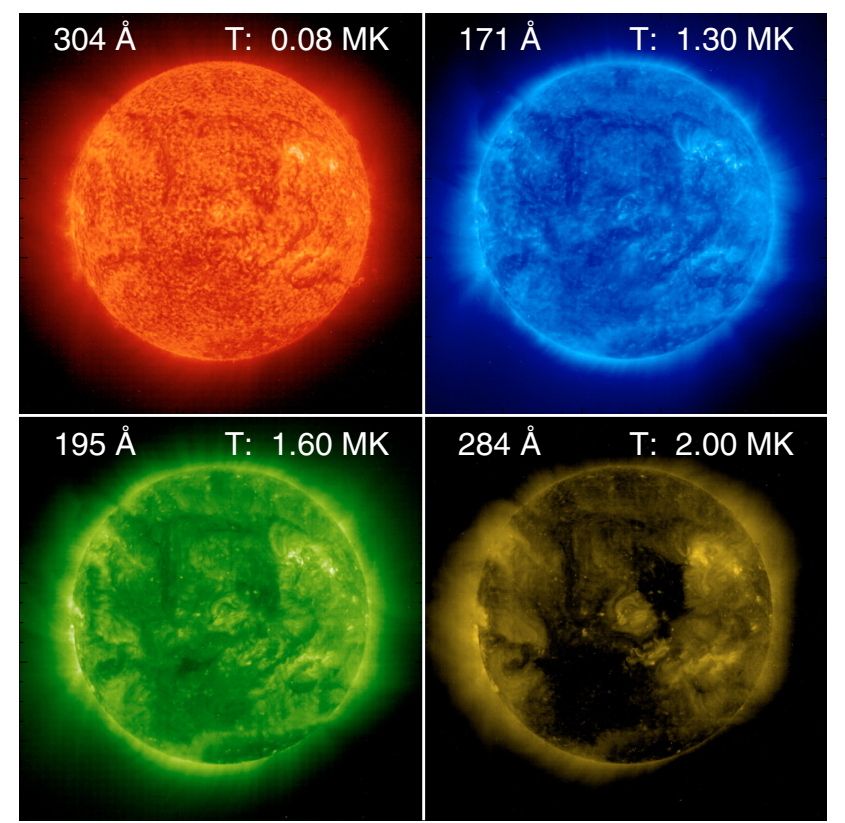

Fig. 2. Composite of four full-disk EIT/SOHO images obtained in the wavelength bands $304 \AA, 171 \AA, 195 \AA$, and $284 \AA$, on 2003 February 24 around 19:00 UT. This was the time of lowest activity in the period of the RESIK quiet-Sun spectra analyzed in this work.

Table 1. Wavelength intervals used for DEM studies.

\begin{tabular}{ccc}
\hline \hline No & Range $[\AA]]$ & Dominant contributor \\
\hline 1 & $3.40-3.62$ & cont. + K XVIII 2p+sat. \\
2 & $3.62-3.80$ & cont. + Ar XVIII 2p, S XVI 4p, 5p \\
3 & $3.85-4.10$ & cont. + Ar XVII 2p+sat, S XV 4p \\
4 & $4.10-4.25$ & cont. + S XV sat. \\
5 & $4.35-4.43$ & cont. + S XV 3p+sat \\
6 & $4.43-4.52$ & cont. + Cl XVI 2p+sat \\
7 & $4.68-4.74$ & cont. + S XVI 2p, + Si XIV 8p \\
8 & $4.74-4.81$ & cont. + Si XIV 6p \\
9 & $5.00-5.13$ & S XV 2p+sat + cont. \\
10 & $5.26-5.35$ & Si XIII 5p+sat. + cont. \\
11 & $5.36-5.50$ & Si XII 4p+sat. + cont. \\
12 & $5.50-5.63$ & Si XII sat. + cont. \\
13 & $5.64-5.72$ & Si XIII 3p + cont. \\
14 & $5.73-5.86$ & Si XII sat. + cont. \\
15 & $5.90-6.00$ & continuum \\
\hline
\end{tabular}

active regions. In Fig. 3, the average of all the 312 spectra is shown, with spectral lines or their expected wavelengths identified. The background is a true solar continuum. Identification of main contributing lines is provided in Table 1.

For these quiet-Sun spectra, there is practically no line emission in channel 1 (shown in blue), though for flare spectra, the prominent triplet of lines due to K XVIII (3.53-3.57 $\AA$ ) is always present (Sylwester et al. 2006b). Similarly, the Ar XVII (3.95-4.00 ̊) triplet in channel 2 (dark red) is barely distinguished but the lines are very prominent during flares. Only weak line emission is visible in channel 3 (orange), identifiable with dielectronic satellites to the parent $1 \mathrm{~s}-3 \mathrm{p}(w 3)$ transition of the H-like S XVI ion. However, channel 4 (yellow) is rich in strong lines formed by relatively cool plasma $(T<\sim 5 \mathrm{MK})$.

The He-like S XV line triplet (5.05-5.10 $\AA$ ) is prominent but with only two components resolved owing to increased line widths. More prominent line features correspond to Si XIII transitions $1 \mathrm{~s}^{2}-1 \mathrm{~s} 4 \mathrm{p}$ and $1 \mathrm{~s}^{2}-1 \mathrm{~s} 3 \mathrm{p}(w 4, w 3)$ at 5.40 and $5.68 \AA$, with nearby Si XII dielectronic satellites at 5.56 and $5.82 \AA(d 4, D)$. The $w 4, d 4$ lines are marked by red arrows, the $w 3, D$ lines by blue arrows. Ratios of the flux in an Si XII dielectronic satellite feature to that in the parent Si XIII resonance line is very sensitive to temperature in the range 2-5 MK (Phillips et al. 2006). Here we used the ratio $D / w 3$ to determine the cooler plasma component temperature characterizing the general coronal emission that the selected RESIK spectra are sensitive to. To do this, we fitted Gaussian profiles to the lines (continuum level subtracted) and the flux ratios compared with theoretical values. The derived "isothermal" temperatures $\left(T_{D / w 3}\right.$ raw $)$ are given in Table 2 for each activity class. The temperatures range from $3.1 \mathrm{MK}$ to $\sim 3.8 \mathrm{MK}$ for the lowest and highest activity classes. They are much lower than those corresponding to flare temperatures (Phillips et al. 2006), even those late in the decay phase. It is striking to see that the intensity of the satellite line $D$ is much higher than the parent line $w 3$, even for a medium- $Z(Z=14)$ element like Si. Generally, this has been seen in flare spectra only for Fe XXIV satellites near Fe XXV lines (near 1.9 $\AA$ : $Z$ for $\mathrm{Fe}=26$ ): note that the flux ratio scales as $Z^{4}$.

A simple way of deducing the physical characteristics of an emitting plasma is by the ratio of fluxes in broad-band filters. For RESIK, the corresponding technique is to take the flux ratio of the total amount of emission in channels 3 and 4. An isothermal assumption is often too crude to be useful (as will be shown later), and physical interpretation of derived values can be ambiguous (Sylwester 1990). The results of the analysis are given in Table 2 (denoted by " $3 / 4$ " entries). The $T_{3 / 4}$ values clearly decrease with increasing activity levels, against expectation, whereas the values of $T_{D / w 3}$ increase. This can be understood in terms of the DEM analysis presented later. The most commonly used diagnostics of physical conditions in the coronal thermal plasma component rely on interpreting the flux ratios measured in the two standard GOES bands. This standard approach (available, e.g., in the GOES section of the IDL SolarSoft package: Freeland \& Handy 1998) was used here, and values of temperatures and emission measures are given in Table 2 as averages over corresponding activity groups (denoted by subscript $\mathrm{G}$ ). It should be stressed, however, that the GOES values derived for the lower intensity classes may be somewhat biased by problems intrinsic to measurements of very low flux levels. The results shown in Table 2 indicate that $T_{\mathrm{G}}$ decreases with activity class, as with the values of $T_{3 / 4}$. With average values of $T$ and $E M$, we may estimate the total thermal energy in the soft X-ray emitting component. It has been shown by Sylwester et al. (2008) that this energy content can be expressed as

$$
E_{\mathrm{th}}=3 k T N_{\mathrm{e}} V=\sqrt{V} T h M \quad \text { erg }
$$

where $V$ is the emitting volume and $T h M$, a "thermodynamic measure", is defined by

$$
T h M=3 k T \sqrt{E M} \quad \mathrm{~g} \mathrm{~cm}^{1 / 2} \mathrm{~s}^{-2} .
$$

The use of $T h M$ is convenient as its variation directly reflects the changes of thermal energy, provided the emitting volume does not change substantially. Derived values of $T h M$ are given in Table 2. It is seen that, in spite of significant differences between the values of $T_{3 / 4}$ and $T_{\mathrm{G}}$ as well $E M_{3 / 4}$ and $E M_{\mathrm{G}}$, values of $T h M_{3 / 4}$ and $T h M_{\mathrm{G}}$ agree quite well. This is an indirect illustration that the value of $T h M$ is relatively independent of the uncertainties in determinations of $T$ and $E M$ (the uncertainties cancel in the product). 


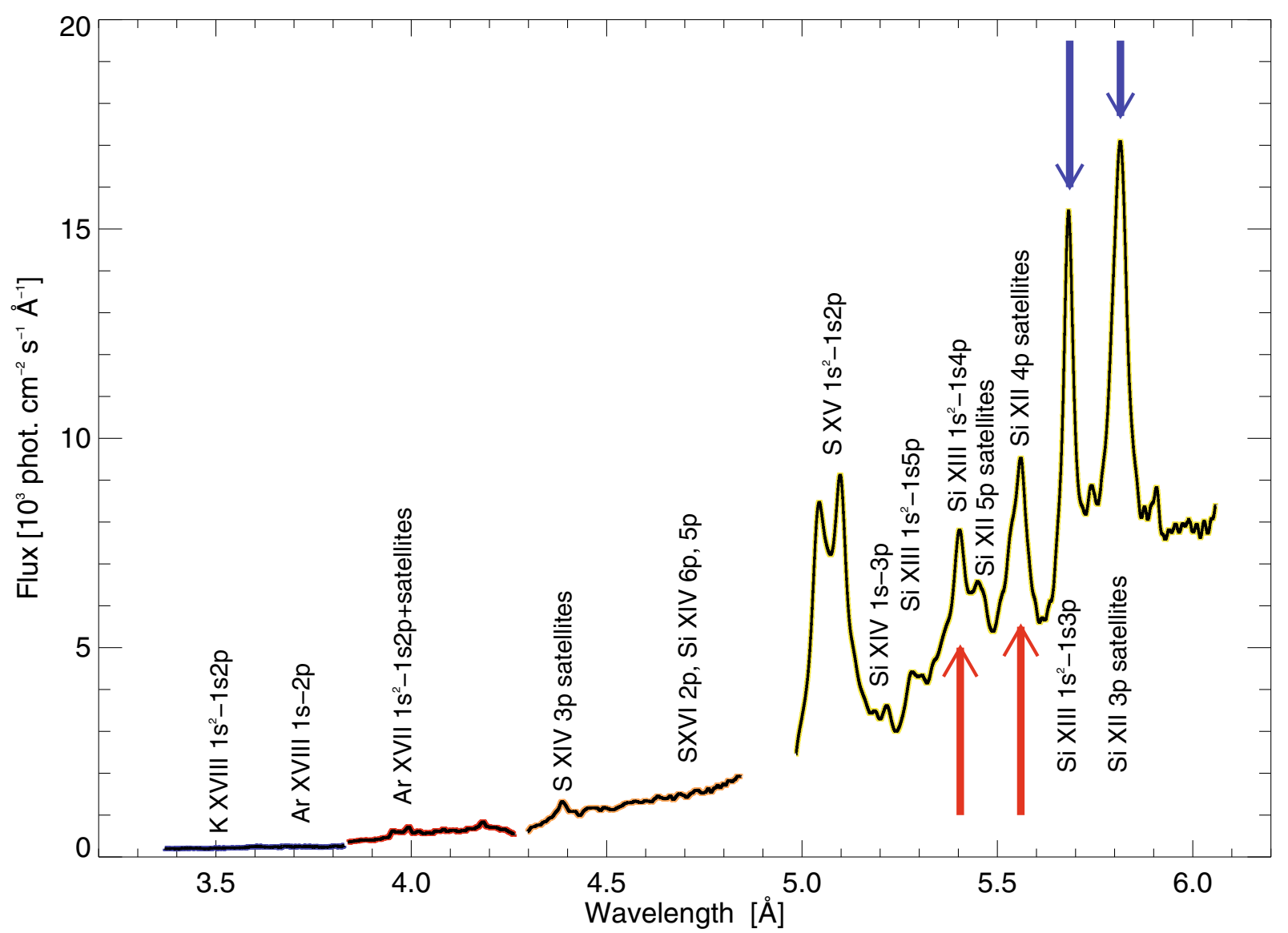

Fig. 3. The absolute RESIK spectrum averaged over 312 quiet Sun intervals selected between 2003 January and March. The most prominent lines are identified.

Table 2. Derived plasma parameters for individual activity classes.

\begin{tabular}{cccccc}
\hline \hline Level & A9-B1 & B1-B2 & B2-B3 & B3-B4 & B4-B5 \\
\hline Isothermal & results & & & & \\
$T_{\mathrm{D} / \mathrm{w} 3}[\mathrm{MK}]$ & 3.11 & 3.68 & 3.84 & 3.88 & 3.85 \\
\hline$T_{\mathrm{G}}[\mathrm{MK}]$ & 7.7 & 6.5 & 5.7 & 5.1 & 5.1 \\
$E M_{\mathrm{G}}{ }^{a}$ & 0.98 & 2.7 & 4.8 & 9.8 & 12.6 \\
$T h M_{\mathrm{G}}{ }^{b}$ & 1.00 & 1.40 & 1.67 & 2.08 & 2.38 \\
\hline$T_{3 / 4}[\mathrm{MK}]$ & 4.8 & 4.8 & 4.0 & 3.6 & 3.8 \\
$E M_{3 / 4}{ }^{a}$ & 1.6 & 7.5 & 19.7 & 47.8 & 59.7 \\
$T h M_{3 / 4}{ }^{b}$ & 0.80 & 1.70 & 2.33 & 3.29 & 3.81 \\
\hline Multithermal & results & & & & \\
El. abun & $10^{-6} \Downarrow$ & & & & \\
Ar & 4.65 & 4.59 & 4.35 & 4.45 & 4.27 \\
$\mathrm{~S}$ & 5.62 & 10.6 & 14.3 & 17.6 & 17.8 \\
$\mathrm{Si}$ & 27.4 & 25.5 & 26.5 & 25.4 & 27.7 \\
\hline$T_{\mathrm{L}}[\mathrm{MK}]$ & 2.9 & 2.6 & 2.5 & 2.4 & 2.3 \\
$E M_{\mathrm{L}}{ }^{a}$ & 0.17 & 0.79 & 2.77 & 8.63 & 22.2 \\
$T h M_{\mathrm{L}}{ }^{b}$ & 1.58 & 3.08 & 5.54 & 9.25 & 14.1 \\
\hline$T_{\mathrm{H}}\left[\mathrm{MK}^{2}\right]$ & 9.1 & 8.0 & 6.9 & 6.5 & 6.1 \\
$E M_{\mathrm{H}}{ }^{a}$ & 0.023 & 0.089 & 0.42 & 1.03 & 1.86 \\
$T h M_{\mathrm{H}}{ }^{b}$ & 0.18 & 0.32 & 0.58 & 0.87 & 1.11 \\
\hline
\end{tabular}

Notes. ${ }^{(a)}$ In $10^{47} \mathrm{~cm}^{-3} ;{ }^{(b)}$ in $10^{15} \mathrm{~g} \mathrm{~cm}^{1 / 2} \mathrm{~s}^{-2}$.

\section{Differential emission measure determinations}

It is generally known that the isothermal approach to determining the characteristics of emitting plasma has serious limitations (Sylwester 1990) and a more advanced approach relies on the concept of differential emission measure, DEM
(Sylwester et al. 1980). We used the DEM approach to analyze of quiet-Sun RESIK spectra arranged by activity grouping. The algorithm we used follows the Bayesian approach, described in detail by Sylwester et al. (1980), and called the WithbroeSylwester algorithm. To apply this algorithm to the analysis of RESIK data, we selected 15 spectral intervals in all four RESIK channels. The wavelength ranges of channels 1 and 2 were divided into 2 broad ranges (between $0.15 \AA$ and $0.25 \AA$ wide) with the remaining 11 ranges in channels 3 and 4 placed around stronger lines or line groups. The ranges are given in Table 1, together with principal contributors to the emission. We performed tests by slightly varying the band widths to find which were the most suitable.

The spectral fluxes were then integrated over each band to improve the count statistics. These fluxes were then used as input to the DEM iterative algorithm. Theoretical spectra providing another source of input for the DEM inversions have been calculated from the CHIANTI v5.2 atomic code (SolarSoft) using the coronal, photospheric, and the other suitable plasma composition models.

The goodness of the fit between the observed and DEMfitted band fluxes was determined by the standard normalized $\chi^{2}$ values,

$\chi^{2}=\frac{1}{n} \sum_{i=1}^{n} \frac{\left(F_{i}-F_{i c}\right)^{2}}{\sigma_{i}^{2}}$,

which is easy to determine when the uncertainties (due to photon count rate statistics) are known. The absolute fluxes should have very small uncertainties because the RESIK intensity calibration is well known (Sylwester et al. 2005). The DEM iterative 


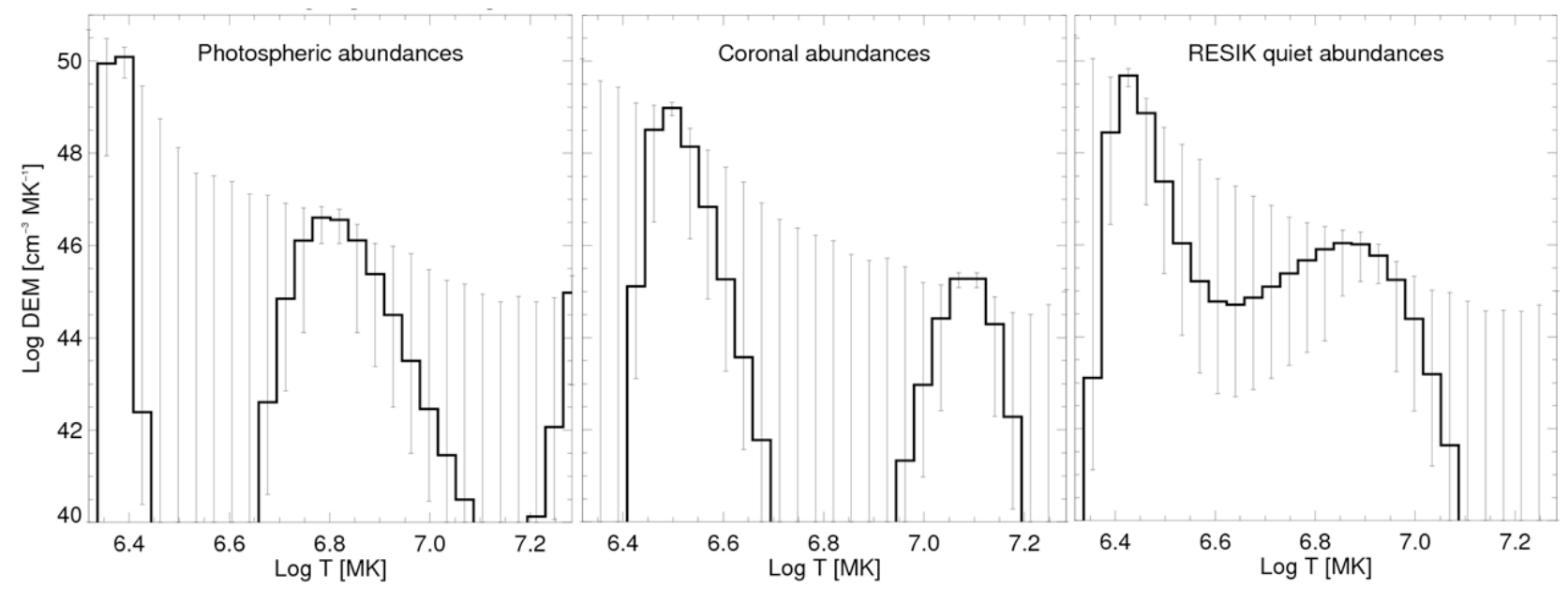

Fig. 4. The differential emission measure distribution calculated from the fluxes in 15 passbands of the averaged nonflaring RESIK spectrum. The results are shown for assuming photospheric (left) coronal (middle) and optimized for the observed spectrum (right) quiet elemental abundances.

process was continued for $10^{4}$ iterations or whenever continuous convergence was attained. In Eq. (3), $F_{i}$ is the photon flux measured in band $i, F_{i c}$ is the flux calculated based on given DEM model, $n$ the number of spectral bands used $(n=15)$, and $\sigma_{i}$ the uncertainty in the measured flux of $i$ band. We have initially performed the DEM inversion over the temperature range from $2 \mathrm{MK}$ to $30 \mathrm{MK}$, but we found it unnecessary to extend the range beyond $T>15 \mathrm{MK}$. No further improvement in the fit quality is observed with a larger band-width in the case of the quiet spectra analysis. Clearly the line and continuum fluxes depend on the plasma composition of the source region, so an appropriate element abundance model must be used to recover the DEM shape accordingly.

It is not obvious which of the two generally used composition models (coronal or photospheric abundances) should be used in the analysis of the quiet corona spectra, so we did the DEM inversions for both sets of plasma abundances. The resulting best-fit DEM shapes for the averaged spectrum (integration time $26.2 \mathrm{~h}$ ) are shown in the left and middle panels of Fig. 4. The envelope of uncertainties of the DEM shape, from 100 Monte-Carlo runs, is shown by the lengths of the error bars. It can be seen how sensitive the resulting shape of the average DEM is to the assumed model of the plasma composition. The best-fit $\chi^{2}$ values are beyond an acceptable range for both photospheric or coronal composition models. We thus concluded that to provide reliable estimates of the DEM shape we should also finely adjust the plasma composition in order to optimize the fit between the observed and calculated spectral band fluxes. To do this, we generated a large spectral look-up, many-dimensional spectral database containing the synthesized profiles in the spectral ranges covered by RESIK (each $0.001 \AA$ ), in 101 temperature points (equidistant between $6<\log T<8$ ), with varying abundances of the elements Ar, S, and Si. The abundances of the other elements not directly influencing the spectral shape for the considered $T$-range of calculations were kept equal to their coronal values. The range of particular element variability was taken from 0.1 to 20 times the coronal abundance value.

With such a large spectral look-up cube it has been relatively easy to look for effects of the dependence of the DEM inversion on particular-element composition. The results obtained are illustrated in Fig. 5, where we show the trend in the minimum value of $\chi^{2}$ as a function of element absolute abundance for Ar, S, and Si. A well-defined minimum is present in each
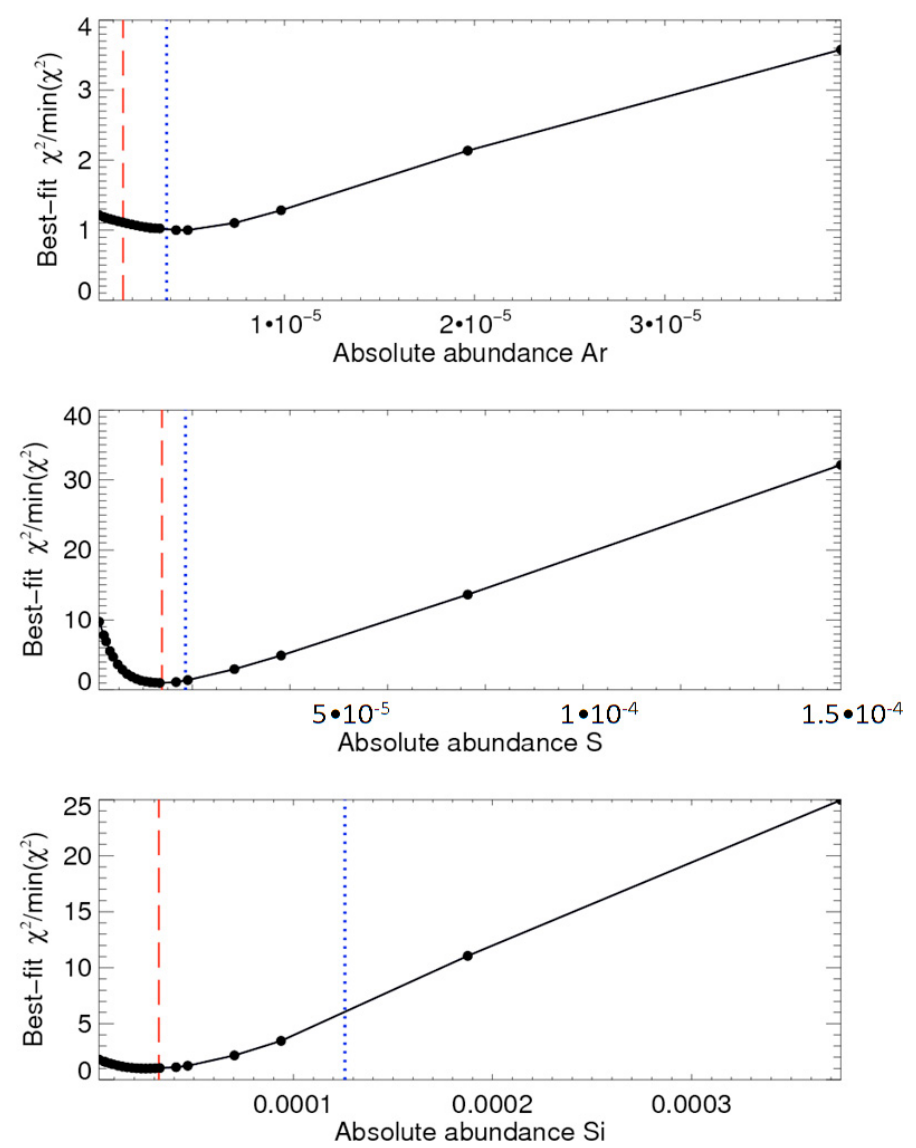

Fig. 5. Ratios of $\chi^{2} / \min \left(\chi^{2}\right)$ plotted against the absolute elemental abundance for Ar, S, and Si. The vertical blue dotted line is plotted at the value corresponding to the respective coronal abundance of the element, while the red dashed at the photospheric value.

of the plots. The location of the minimum along the abundance axis is placed close to the photospheric value for $\mathrm{S}$ and $\mathrm{Si}$. For the element Ar, which has a high value of first ionization potential (FIP), derived optimum abundance is above the coronal value. It follows that neither the photospheric nor the coronal abundance models can be used to describe the observed set of spectral intensities at the same time. Therefore we performed 


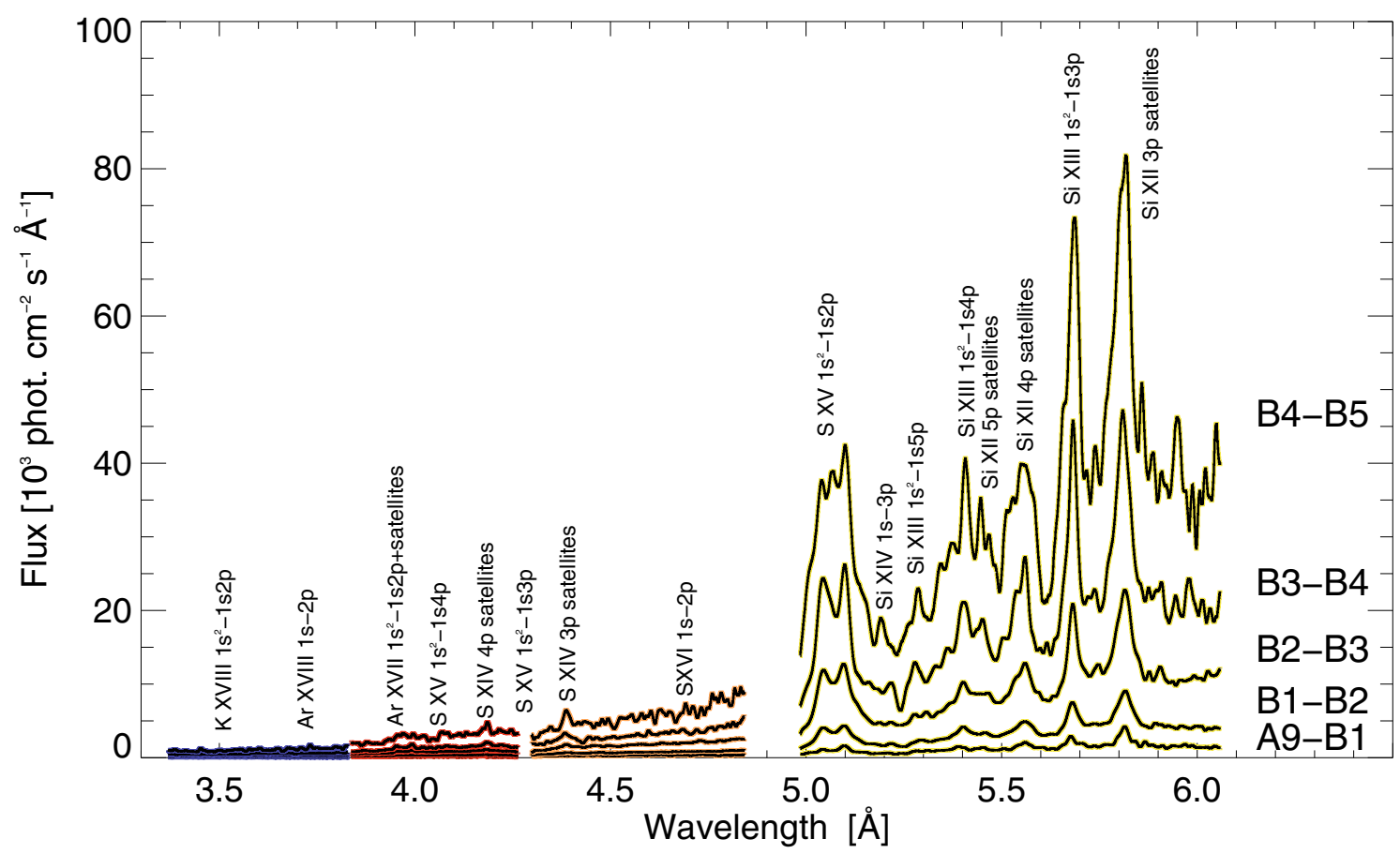

Fig. 6. The absolute RESIK spectra in linear scale grouped according to the GOES class. The most prominent lines are indicated.

the DEM inversion using the optimum abundance values as determined from the position of the minima in Fig. 5. The result is shown in Fig. 4 (right panel). It is seen that the calculated shape of the DEM is smoother in comparison with the photospheric or coronal cases and the error envelope decreases. The calculated value of the normalized $\chi^{2}$ is also brought now into an acceptable range. As a consequence of this exercise, we decided to perform the composition optimization process for every DEM inversion for corresponding activity class. The results are shown in Table 2 and illustrated in Fig. 7.

The calculated, abundance-optimized shapes of DEM distribution are presented in different colors. The black histogram represents the highest activity class (B4-B5). No error bars are shown in order to increase the visibility. It is observed that all DEM distributions are bimodal with the cooler (lower temperature) and hotter (higher temperature) components well separated. As the activity level increases, the average temperatures of the low and high- $T$ components shift toward lower temperatures. This is somewhat unexpected but can be understood as partly due to the deconvolution process working more effectively when the emission measure ratio of the lower and higher- $T$ components is decreasing. The amount of cooler plasma found is orders of magnitude higher than the hotter one. Depending on the activity level, this amounts to 750 and 1190 for the lower and higher activity levels, respectively. In Table 2 the basic characteristics of the cooler (index L) and hotter (index H) plasma components is presented. In calculating the total thermal energy content from the obtained DEM $=\varphi(T)$ distributions, we used the following formulas, derived assuming a constant pressure or density in the emission volume:

$$
\begin{aligned}
& \left.E_{\mathrm{th}}\right|_{p=\text { const. }}=\sqrt{V} 3 k \sqrt{\int T^{2} \varphi(T) \mathrm{d} T} \\
& \left.E_{\mathrm{th}}\right|_{N e=\text { const. }}=\sqrt{V} 3 k \frac{\int T \varphi(T) \mathrm{d} T}{\sqrt{\int \varphi(T) \mathrm{d} T}} .
\end{aligned}
$$

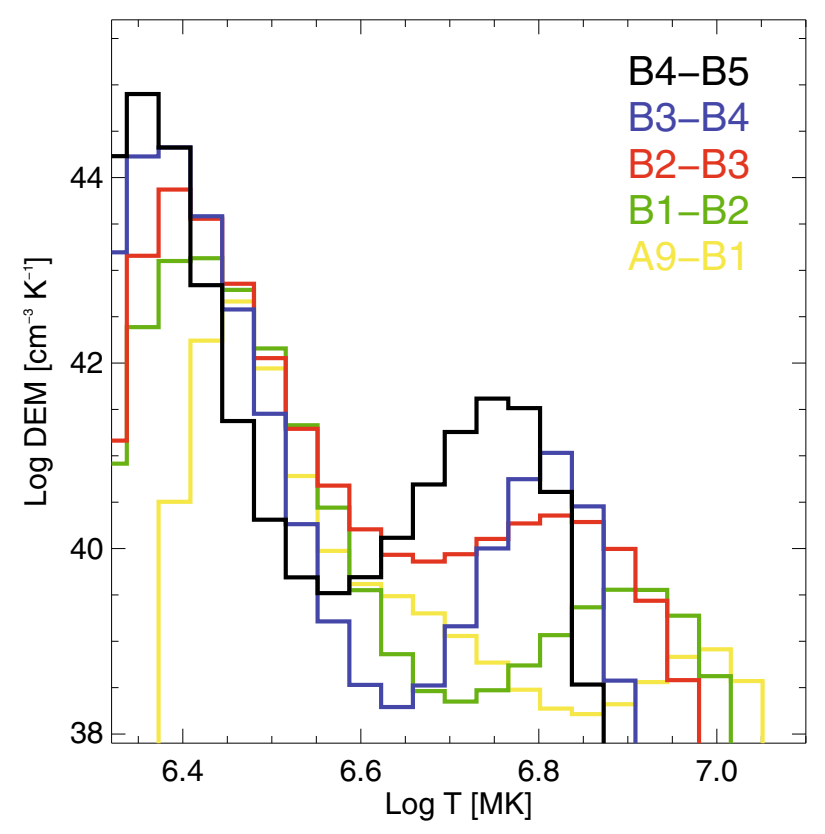

Fig. 7. The histogram representation of differential emission measure (DEM) distributions as obtained from RESIK spectra for individual classes of solar activity. Different colors correspond to the following classes: black (dark) represents the B4-B5 class and yellow (lightest) A9-B1 class.

It is straightforward to assign the meaning of thermodynamic measure $T h M$ to the righthand factors of Eqs. (4) and (5). It was found that the difference between results obtained in the constant pressure or constant density assumptions did not differ by more than a few percent for the considered DEM shapes, so in Table 2 we insert average values, characteristic of the cooler and hotter components. With the knowledge of respective volumes occupied by the cooler and the hotter plasma, it would be possible to 
estimate thermal energy content of respective components. This study is in progress.

The results of DEM calculations with the elementabundance optimization for the five activity classes are shown in Fig. 7 in different colors. The bimodal character of resulting DEM is evident. The average values of the parameters characterizing each of the two plasma components are given in Table 2 along with the associated set of optimum elemental abundances is provided. The data set in Table 2 shows that the abundances of Ar and $\mathrm{Si}$ do not depend on the activity level, but the sulfur abundance of the plasma contributing to RESIK channel 4 spectra systematically increases by a factor of $\sim 4$ from a level well below the photospheric to the coronal abundance with activity increasing from lower to higher classes. The reason for this behavior is not known at present. It is important to note that the optimum abundances of $\mathrm{Si}$ are below the generally assumed photospheric level, but the Ar abundances (Ar is a high-FIP element) lie even above coronal values. As concerns the energy content associated with the two plasma components, it is interesting to note that the thermodynamic measure ratios of the lower and higher- $T$ components are much less than the ratio of respective emission measures. Provided that the emitting volumes are comparable, this would mean that the ratio of the total thermal energy content of the hotter and cooler components is not all that different, and as a consequence, the heating processes responsible for formation of the hotter component should not be disregarded in studies of the overall energy balance even for nonflaring coronal conditions.

\section{Concluding remarks}

We analyzed 312 individual RESIK spectra grouped into 5 different activity classes based on GOES levels of activity recorded at the time of the spectra collection. All selected spectra were taken during the nonflaring, low activity conditions prevailing in the corona, though some weak active-region emission was always present. The spectral observations (all with a five-minute integration time) cover the period between 2003 January 1 and March 14. The analysis assumed both isothermal and multithermal distributions, the latter leading to determination of DEM distributions for each activity class. The DEMs were obtained with the Withbroe-Sylwester Bayesian iterative, maximum likelihood procedure. The fluxes integrated over 15 wavelength bands covering the range 3.3-6.1 $\AA$ were used as an input data for the deconvolution. We found it necessary to allow for the emitting plasma composition to be nonstandard, i.e. neither coronal nor photospheric, by varying the abundances of those elements making important contributions to the line emission in the spectra. This analysis has never been done before except for a few flares (to be published, Montreal COSPAR). The main results of the present study follow.

1. It is necessary to use the multithermal approach in analyzing the spectra. The results obtained in the isothermal approximation provide different values of $T$ and $E M$ parameters depending on the ratio considered. However, the values of socalled thermodynamic measure that is directly related to the total thermal energy plasma content are very close, because they are surprisingly independent of the particular ratio used for its determination.

2. It appears necessary to allow for the plasma composition differences when using the multitemperature approach in the analysis. Neither coronal nor photospheric composition models are able to describe the observed spectra satisfactorily. This result may also bias the outcome of any isothermal analysis performed with an isothermal approach.

3. The two-temperature character of the DEM shape determined here for non-flaring plasmas has also been obtained for flares (Sylwester et al. 2008). The presence of the higher$T$ component, with $T$ somewhat below $10 \mathrm{MK}$, is physically important. The emission measure associated with this hotter plasma is $\sim 3$ orders of magnitude smaller than in the generally accepted $T \sim 2-3 \mathrm{MK}$ component. This higher- $T$ component is required because it is impossible to reproduce the observed spectra without it.

It is worth noting that the presence of a hotter component in active region emission has been recently suggested by Reale et al. (2009) from their analysis of Hinode XRT images of an active region and by Schmelz et al. (2009), although the very high temperature of the hotter component discussed by Schmelz et al. (2009) has been reduced to a value near what is obtained in this work (Schmelz 2009, priv. comm.).

Acknowledgements. RESIK is a common project between the NRL (USA), MSSL and RAL (UK), IZMIRAN (Russia), and SRC (Poland). This work was partially supported by the International Space Science Institute in the framework of an international working team (No. 108). We acknowledge the travel support from a UK Royal Society/Polish Academy of Sciences International Joint Project. CHIANTI is a collaborative project involving the NRL (USA), RAL (UK), MSSL (UK), the Universities of Florence (Italy) and Cambridge (UK), and George Mason University (USA). The research leading to these results received partial funding from the European Commission's Seventh Framework Programme (FP7/2007-2013) under grant agreement No. 218816 (SOTERIA project, www. soteria-space.eu).

\section{References}

Brosius, J. W., Davila, J. M., \& Thomas, R. J. 1996, ApJS, 106, 143 Brosius, J. W., Davila, J. M., \& Thomas, R. J. 1998, ApJS, 119, 255 Chifor, C., del Zanna, G., Mason, H. E., et al. 2007, A\&A, 462, 323 Curdt, W., Landi, E., \& Feldman, U. 2004, A\&A, 427, 1045

Delaboundiniere, J.-P., Artzner, G. E., Brunaud, J., et al. 1995, Sol. Phys., 162, 291

Dupree, A. K., \& Reeves, E. M. 1971, ApJ, 165, 599

Dupree, A. K., Huber, M. C. E., Noyes, R. W., et al. 1973, ApJ, 182, 321

Freeland, S. L., \& Hardy, B. N. 1998, Sol. Phys., 182, 497

Hannah, I. G., Hurford, G. J., Hudson, H. S., Lin, R. P., \& van Bibber, K. 2007, ApJ, 659, L77

Klimchuk, J. A., Patsourakos, S., \& Cargill, P. J. 2008, ApJ, 682, 1351

Kretzschmar, M., Lilensten, J., \& Aboudarham, J. 2004, A\&A, 419, 345

Malinovsky, M., \& Heroux, L. 1973, ApJ, 181, 1009

Peterson, L. E., Schwartz, D. A., Pelling, R. M., \& Mckenzie, D. 1966, J. Geophys. Res., 71, 5778

Phillips, K. J. H., Dubau, J., Sylwester, J., \& Sylwester, B. 2006, ApJ, 638, 1154

Ralchenko, Yu., Feldman, U., \& Doschek, G. A. 2007, ApJ, 659, 1682 Reale, F., Testa, P., Klimchuk, J. A., \& Parenti, S. 2009, ApJ, 698, 756 O'Shea, E., Gallagher, P. T., Mathioudakis, M., et al. 2000, A\&A, 358, 741 Schmelz, J. T., Saar, S. H., DeLuca, E. E., et al. 2009, ApJ, 693, L131 Sylwester, B., Sylwester, J., Siarkowski, M., et al. 2006a, Adv. Space Res., 38, 1534

Sylwester, B., Sylwester, J., Kepa, A., et al. 2006b, Sol. System Res., 40, 125 Sylwester, B., Sylwester, J., \& Phillips, K. J. H. 2008, J. Astrophys. Astr., 29, 147

Sylwester, J. 1990, The Dynamic Sun, Proceedings of the 6th European Meeting on Solar Physics, Debrecen, 21-24 May, ed. L. Dezso, Debrecen Heliophysical Observatory of the Hungarian Academy of Sciences, 7, 212 Sylwester, J., Schrijver, J., \& Mewe, R. 1980, Sol. Phys., 67, 285

Sylwester, J., Gaicki, I., Kordylewski, Z., et al. 2005, Sol. Phys., 226, 45 Sylwester, J., Sylwester, B., \& Phillips, K. J. H. 2008, ApJ, 681, L117 Warren, H. P., Mariska, J. T., \& Lean, J. 1998, J. Geoph. Res., 103, 12077 Vernazza, J. E., \& Reeves, E. M. 1978, ApJS, 37, 485

Young, P. R., Del Zanna, G., Mason, H. E., et al. 2007, PASJ, 59, S857 